University of Nebraska - Lincoln

DigitalCommons@University of Nebraska - Lincoln

April 2006

\title{
Finite-temperature anisotropy of magnetic alloys
}

\author{
Ralph Skomski \\ University of Nebraska-Lincoln, rskomski2@unl.edu
}

O.N. Mryasov

University of Nebraska - Lincoln

J. Zhou

University of Nebraska - Lincoln

David J. Sellmyer

University of Nebraska-Lincoln, dsellmyer@unl.edu

Follow this and additional works at: https://digitalcommons.unl.edu/physicssellmyer

Part of the Physics Commons

Skomski, Ralph; Mryasov, O.N.; Zhou, J.; and Sellmyer, David J., "Finite-temperature anisotropy of magnetic alloys" (2006). David Sellmyer Publications. 6.

https://digitalcommons.unl.edu/physicssellmyer/6

This Article is brought to you for free and open access by the Research Papers in Physics and Astronomy at DigitalCommons@University of Nebraska - Lincoln. It has been accepted for inclusion in David Sellmyer Publications by an authorized administrator of DigitalCommons@University of Nebraska - Lincoln. 


\title{
Finite-temperature anisotropy of magnetic alloys
}

\author{
R. Skomski ${ }^{\mathrm{a})}$ \\ Department of Physics and Astronomy, University of Nebraska, Lincoln, Nebraska 68588 \\ and Center for Materials Research and Analysis, University of Nebraska, Lincoln, \\ Nebraska 68588 \\ O. N. Mryasov \\ Department of Physics and Astronomy, University of Nebraska, Lincoln, Nebraska 68588 \\ and Center for Materials Research and Analysis, University of Nebraska, Lincoln, Nebraska 68588 \\ and Seagate Research, 1251 Waterfront Place, Pittsburgh, Pennsylvania 15222-4215
}

J. Zhou and D. J. Sellmyer

Department of Physics and Astronomy, University of Nebraska, Lincoln, Nebraska 68588, and Center for Materials Research and Analysis, University of Nebraska, Lincoln, Nebraska 68588

(Presented on 1 November 2005; published online 27 April 2006)

The temperature dependence of the magnetic anisotropy of ferromagnetic materials is analyzed. Simple ferromagnets, such as Fe and Co, obey the $m=n(n+1) / 2$ power laws predicted by the Callen and Callen [Phys. Rev. 129, 578 (1963)] theory, but in alloys, the applicability of the theory is an exception rather than the rule. Many alloys, such as the rare-earth transition-metal intermetallics and $\mathrm{L}_{0}$ magnets, violate a basic assumption of the theory, namely, that the single-ion anisotropy and the spontaneous magnetization have the same origin. This is the reason for significant deviations from the Callen and Callen behavior, such as the $m=2$ law we obtained for $\mathrm{L} 1_{0}$ alloys. (C) 2006 American Institute of Physics. [DOI: 10.1063/1.2176892]

\section{INTRODUCTION}

Controlling the temperature dependence of the magnetic anisotropy is a key requirement in the development and improvement of magnetic materials. Permanent magnets often require a high anisotropy constant $K_{1}$ above room temperature or a zero temperature gradient $d K_{1} / d T$ at the operation temperature. In recording media, the emphasis is on wellcontrolled finite-temperature anisotropy, in order to combine thermal stability with writability. Since magnetocrystalline anisotropy is characterized by a pronounced temperature dependence, ${ }^{1-3}$ this is a formidable challenge, and the theoretical description of the magnetic anisotropy has remained a nontrivial and partially controversial issue. ${ }^{4-7}$

One question is the applicability of the Callen and Callen theory, ${ }^{8}$ which expresses the temperature dependence of the anisotropy by a power law

$$
K(T)=K(0)\left(\frac{M_{s}(T)}{M_{s}(0)}\right)^{m}
$$

where the $n$th order anisotropy constants obey $m=n(n$ $+1) / 2$. In particular, for cubic and uniaxial magnets, the respective exponents are $m=3$ and $m=10$. The popular theory, which goes back to Akulov, ${ }^{9}$ has been used as a starting point for the theoretical ${ }^{6}$ and experimental ${ }^{10}$ discussion of a broad range of magnetic materials. However, it is at odds with the experiment and with other models. ${ }^{1-5,7}$ For example, the single-ion anisotropy of $\mathrm{L}_{0}$ magnets obeys a power law with $m=2 .^{5}$

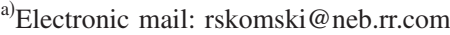

In this paper, we reexamine the basic assumptions of the Callen-Callen approach to single-ion anisotropy and show that its applicability is an exception. We discuss cases of single-ion anisotropy where the Callen-Callen theory fails completely, and then analyze the $\mathrm{L} 1_{0}$ magnets, which have recently attracted renewed interest as materials for ultrahighdensity magnetic recording.

\section{QUANTUM-MECHANICAL BACKGROUND}

In most magnetic materials, the main source of anisotropy is magnetocrystalline anisotropy, which reflects the competition between the spin-orbit coupling and crystal-field interaction. The crystal field, which contains both electrostatic and hopping contributions, modifies the orbits of the electrons and translates, via spin-orbit coupling, into magnetic anisotropy. ${ }^{3}$

Magnetocrystalline anisotropy is, essentially, a singleion property, realized by embedding the atom in a metallic or nonmetallic crystalline environment. ${ }^{3}$ This must be compared to the Néel model, ${ }^{11}$ which ascribes the anisotropy to explicit pair interactions. Even in itinerant magnets, where the moment formation involves many atoms, the anisotropy is realized by the intra-atomic spin-orbit coupling. The role of the crystalline environment is to realize the crystal field and to stabilize the anisotropy against thermal excitations.

In a simple classical picture, the anisotropy energy of a magnetic atoms is proportional to $3 \cos ^{2} \theta-1$, where $\theta$ is the angle between the crystallographic $c$ axis and the direction of the atom's magnetic moment. Quantum-mechanical generalizations of this expression, such as $3 S_{z}^{2}-S(S+1)$, yield quantitative corrections rather than qualitative changes. Intra- 


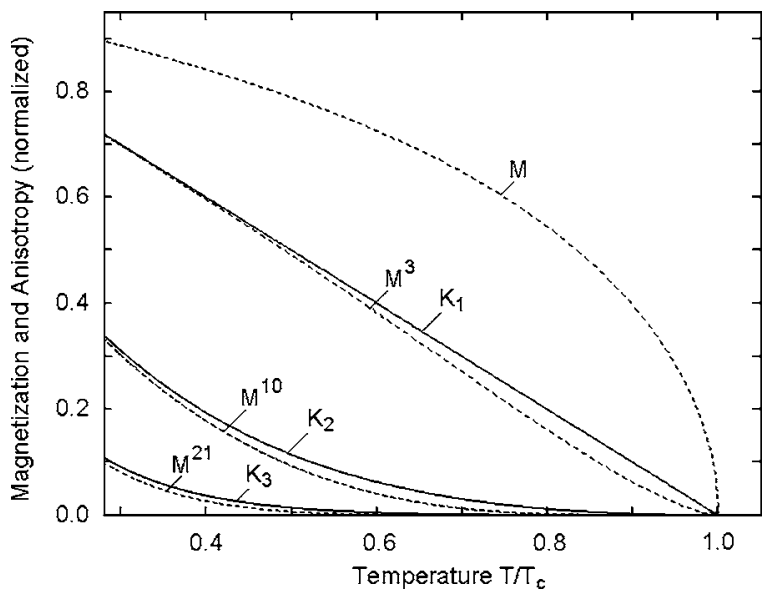

FIG. 1. Predictions for the classical Heisenberg model. The figure compares the Callen and Callen results (dashed) with the corresponding exact solutions (solid). Note that the deviations from the power-law behavior are unrelated to critical fluctuations.

atomic finite-temperature excitations where $\left|S_{z}\right|<S$ are the main reason for the temperature dependence of the anisotropy. ${ }^{1,3}$

\section{SIMPLE FERROMAGNETS}

For one-sublattice magnets, such as $\mathrm{Fe}$ and $\mathrm{Co}$, the Callen and Callen law is a reasonable approximation. Let us consider the classical Heisenberg (or $S=\infty$ ) mean-field model with uniaxial anisotropy, where a normalized classical spin $\mathbf{s}$ interacts with a selfconsistent mean field $\mathbf{H}_{\text {eff }}=J m \mathbf{e}_{z}$ and $m$ $=\left\langle s_{z}\right\rangle$ is the self-consistently determined average magnetization. The model yields thermal averages

$$
\left\langle\cos ^{n} \theta\right\rangle=\frac{\int \exp \left(J m / k_{B} T\right) \cos ^{n} \theta \sin \theta d \theta}{\int \exp \left(J m / k_{B} T\right) \sin \theta d \theta},
$$

where both integrals extend from $\theta=0$ to $\theta=\pi$. The quantities of interest in this paper correspond to the Legendre polynomials of $\cos \theta$. For example, $m=\langle\cos \theta\rangle, \quad K_{1}(T)$ $=1 / 2 K_{1}(0)\left(3\left\langle\cos ^{2} \theta\right\rangle-1\right), \quad$ and $\quad K_{2}(T)=K_{2}(0)\left(35\left\langle\cos ^{4} \theta\right\rangle\right.$ $\left.-30\left\langle\cos ^{2} \theta\right\rangle+5\right) / 8$. In general, the Legendre polynomials of the order $n$ describe $n / 2$ th anisotropy constants.

At low temperatures, the model obeys the Callen and Callen relation. In this limit,

$$
\left\langle\cos ^{n} \theta\right\rangle=1-n k_{B} T / J,
$$

so that $m=1-k_{B} T / J$ and $K_{1}(T)=K_{1}(0)\left(1-3 k_{B} T / J\right)$. Using our assumption that $k_{B} T \ll J$, we can rewrite these two equations as $K_{1}(T) / K_{1}(0)=m^{3}$, as expected for a Callen and Callen analysis. Figure 1 compares the Callen and Callen approximation with the exact mean-field solution, as obtained by explicit integration of Eq. (2).

\section{INTERMETALLIC ALLOYS}

A key assumption of the Callen and Callen theory is that the temperature dependence of the magnetization and the temperature dependence of the anisotropy have the same ori-

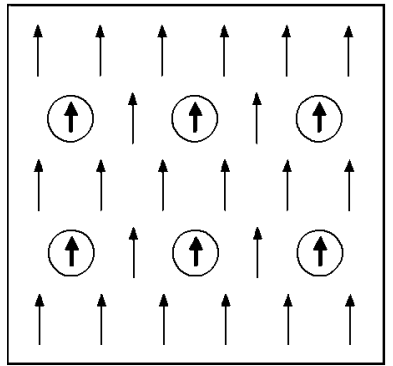

(a)

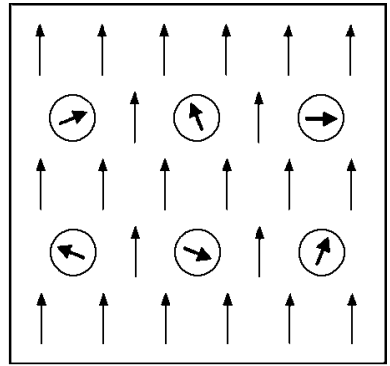

(b)
FIG. 2. Spin structure of transition-metal-rich RE-TM intermetallics: (a) zero temperature and (b) room temperature. The rare-earth atoms (spheres) are embedded in a transition-metal environment. The anisotropy, indicated by the thickness of the arrows, is dominated by the rare-earth atoms. Due to the relatively weak interatomic RE-TM exchange, the anisotropy breaks down well below $T_{c}$.

gin. For example, both $M_{s}(T)$ and $K_{1}(T)$ reflect the misalignment angle $\theta$. However, in most alloys there is no simple relation between the magnetization and the anisotropy. Figure 2 illustrates this point for rare-earth transition-metal (RE$\mathrm{TM}$ ) intermetallics, such as $\mathrm{SmCo}_{5}$ and $\mathrm{Nd}_{2} \mathrm{Fe}_{14} \mathrm{~B}$, where the leading single-ion rare-earth anisotropy contribution of permanent magnet intermetallics scales as $1 / T^{2}{ }^{4}$ Translated into the Callen and Callen picture, rare-earth transition-metal intermetallics exhibit $m \approx 0$. Figure 3 shows $K_{1}(T)$ for a typical RE-TM magnet.

An interesting example is the finite-temperature magnetization of $\mathrm{L} 1_{0}$ magnets. ${ }^{5,10,12}$ Elemental $4 d / 5 d$ magnets, such as Pd and Pt, are exchanged-enhanced Pauli paramagnets, but in a ferromagnetic environment they are easily spin polarized by neighboring $3 d$ atoms. The $4 d / 5 d$ moment contributes little to magnetization and Curie temperature but plays a key role in the realization of magnetic anisotropy. The limiting factor is the itinerant spin polarization of the $4 d / 5 d$ moment. If the $\mathrm{Pt}$ was fully spin polarized, the anisotropy of PtCo would be $20 \mathrm{MJ} / \mathrm{m}^{3}$, as compared to the experimental value of about $5 \mathrm{MJ} / \mathrm{m}^{3} \cdot{ }^{3,14}$ Our analytical single-ion model predicts an $m=2$ law, ${ }^{5,12}$ whereas refined numerical calculations yield $m=2.08 .^{7}$ The latter value amounts to a single sublattice or "Callen and Callen" contribution of the order of $8 \%$.

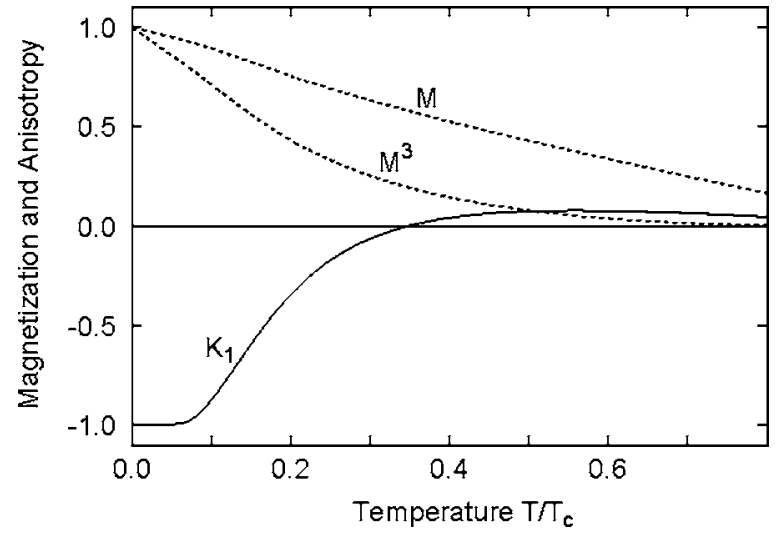

FIG. 3. Violation of the Callen and Callen law in rare-earth transition metallics. The solid line describes, for example, materials such as $\mathrm{NdCo}_{5}$. This illustrates the complete failure of the Callen and Callen theory. 


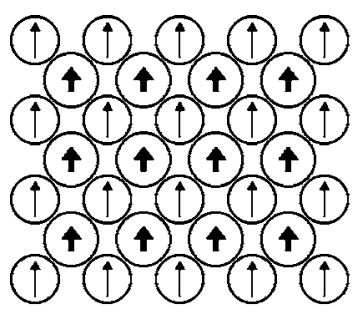

(a)

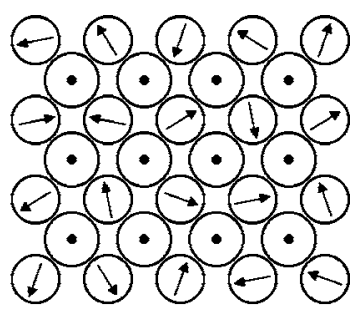

(b)
FIG. 4. Spin structures (schematic): (a) ferromagnetism and (b) paramagnetism. This figure illustrates the physical origin of the temperature dependence of the anisotropy of $\mathrm{L}_{0}$ magnets.

Figure 4 illustrates the atomic origin of the temperature dependence of $\mathrm{L} 1_{0}$ magnets. In the model, the mean-field Hamiltonian $\mathrm{H}=-J m s$ is replaced by an expression $\mathrm{H}(m,\langle S\rangle)$, where $m$ is the $4 d / 5 d$ moment and $\langle S\rangle$ is the average $3 d$ magnetization. The $3 d$ sublattice exerts an exchange field $J^{*}\langle S\rangle$ on the $4 d / 5 d$ sublattice, creates a $4 d / 5 d$ moment and realizes, via spin-orbit coupling, the magnetic anisotropy. The mechanism involves pair interactions (hopping) between the neighboring $3 d$ and $4 d / 5 d$ atoms ${ }^{5}$ but is different from true pair anisotropy, because the main function of the $3 d$ sublattice is to realize a single-ion crystal field. At high temperatures, $\langle S\rangle$ approaches zero, and the anisotropy collapses. Figure 5 shows the temperature dependence of the anisotropy for various types of magnetic compounds.

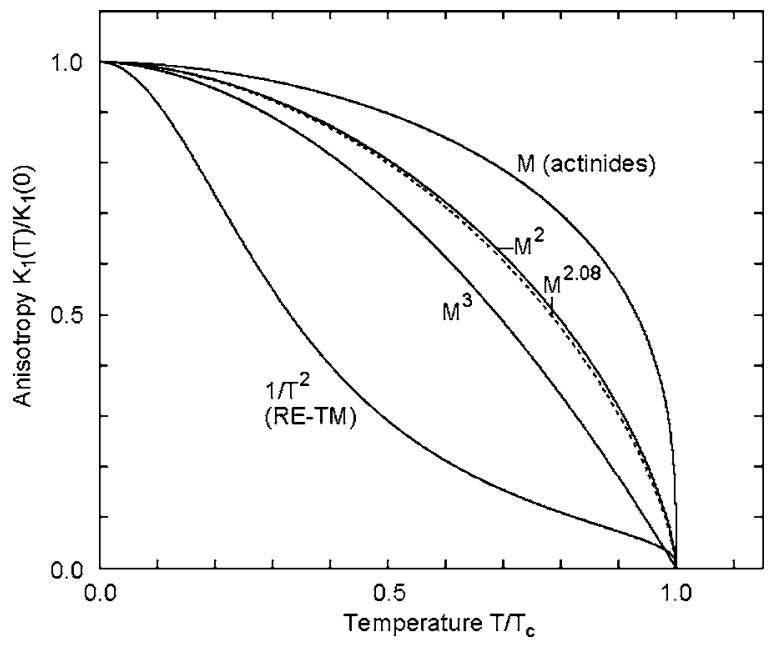

FIG. 5. Temperature dependence of second-order anisotropy constants of some magnetic compounds. Cubic and noncubic actinide magnets exhibit $m=1$ (Ref. 15).

\section{DISCUSSION AND CONCLUSIONS}

Figure 5 shows that the predictions for $m=2$ and $m=3$ are relatively similar to each other and to the experimental findings. ${ }^{10}$ By varying the involved parameters, both approaches yield a fair agreement with experiment. For example, replacing a mean-fieldlike magnetization $M_{\mathrm{o}}$ (1 $\left.-T / T_{c}\right)^{1 / 2}$ by $M_{\mathrm{o}}\left(1-T / T_{c}\right)^{0.33}$, as appropriate for an anisotropic ferromagnet close to $T_{c}$, is sufficient to blur the distinction between the $m=2$ and $m=3$. Furthermore, there are secondary effects such as thermal expansion. We will not dwell on these points but emphasize that the mechanisms beyond $m=2$ and $m=3$ are mutually exclusive and that the crucial involvement of two sublattices speaks in favor of $m=2$.

In the light of the present findings, and of those described in the literature, ${ }^{1,3-5,7}$ it is not surprising that the simulations in Ref. 6 disagree with the Callen and Callen theory. Beyond its original scope, namely, magnets such as $\mathrm{Fe}$ and $\mathrm{Ni}$, deviations from the Callen and Callen power laws are the rule rather than the exception. As we have elaborated in this paper, the main reason is the nontrivial involvement of two or more sublattices.

\section{ACKNOWLEDGMENTS}

This work is partially supported by NSF-MRSEC, the W. M. Keck Foundation, and CMRA. The authors are grateful to R. W. Chantrell for stimulating discussions.

${ }^{1}$ J. F. Herbst, Rev. Mod. Phys. 63, 819 (1991).

${ }^{2}$ K. Kumar, J. Appl. Phys. 63, R13 (1988).

${ }^{3}$ R. Skomski and J. M. D. Coey, Permanent Magnetism (Institute of Physics, Bristol (1999).

${ }^{4}$ R. Skomski, J. Appl. Phys. 83, 6724 (1998).

${ }^{5}$ R. Skomski, A. Kashyap, and D. J. Sellmyer, IEEE Trans. Magn. 39, 2917 (2003).

${ }^{6}$ J. B. Staunton, S. Ostanin, S. S. A. Razee, B. L. Gyorffy, L. Szunyogh, B. Ginatempo, and E. Bruno, Phys. Rev. Lett. 93, 257204 (2004).

${ }^{7}$ O. N. Mryasov, U. Nowak, K. Guslienko, and R. Chantrell, Europhys. Lett. 69, 805 (2005).

${ }^{8}$ E. R. Callen and H. B. Callen, Phys. Rev. 129, 578 (1963).

${ }^{9}$ N. Akulov, Z. Phys. 100, 197 (1936).

${ }^{10}$ N. H. Hai, N. M. Dempsey, and D. Givord, IEEE Trans. Magn. 39, 2914 (2003).

${ }^{11}$ L. Néel, J. Phys. Radium 15, 225 (1954).

${ }^{12}$ R. Skomski, A. Kashyap, and J. Zhou, Scr. Mater. 53, 389 (2005).

${ }^{13}$ T. Klemmer, D. Hoydick, H. Okumura, B. Zhang, and W. A. Soffa, Scr. Metall. Mater. 33, 1793 (1995).

${ }^{14}$ R. A. McCurrie, Ferromagnetic Materials: Structure and Properties (Academic, London, 1994).

${ }^{15}$ R. Skomski, J. Appl. Phys. 91, 8489 (2002). 